\title{
Analysis of particle dynamics in a pin mill by Discrete Element Method
}

\author{
Wei Pin Goh and Mojtaba Ghadiri* \\ School of Chemical and Process Engineering, University of Leeds, Leeds, LS2 9JT, UK
}

\begin{abstract}
Milling is an important process for tailoring the particle size distribution for enhanced attributes, such as dissolution, content uniformity, tableting, etc., especially for active pharmaceutical ingredients and excipients in pharmaceutical industries. Milling performance of particulate solids depends on the equipment operating conditions (geometry, process conditions and input energy etc.) as well as material properties (particle size, shape, and mechanical properties, such as Young's modulus, hardness and fracture toughness). In this paper the particle dynamics in a pin mill is analysed using Discrete Element Method (DEM), combined with a novel approach for assessing particle breakability by single particle impact testing. A sensitivity analysis is carried out addressing the effect of the milling conditions (rotational speed and feed particle flow rate), accounting for feed mechanical properties on the breakage behaviour of the particles. Particle collision energy spectra are calculated and shown to have a distribution with the upper tail end being close to the maximum energy associated with the collision with the rings. Breakage is primarily due to collisions with the rings, except for large particles that are comparable in size with the gap between the rings, nipping is also a contributory breakage mechanism.
\end{abstract}

\section{Introduction}

Milling is commonly used in a wide range of manufacturing operations to tailor desired product specifications and quality attributes [1]. The milling performance of particulates is dictated by the mechanical properties of the material (hardness, fracture toughness and Young's modulus) and the operating conditions of the chosen mill such as the geometry, process conditions, input energy, etc. The use of pin mill is ubiquitous as it has a small footprint, is versatile, able to mill a wide range of solids and has a relatively reasonable energy consumption. Pin mill has essentially two steel plates on which a number of concentric pins are mounted and configured to either (i) have one of the discs rotating while the other stays stationary or (ii) have both of the discs counter- rotating to achieve higher energy impacts. The working principle of a pin mill is similar to that of a hammer mill, where the size reduction is due to the impacts between the particles and the pins but generally at higher tip speed configuration.

At the early stages of product and process development, adequate test material is not generally available. Therefore, a methodology is required to predict the milling behaviour based on the properties of the test material. For materials that exhibit semi-brittle behaviour, the case under consideration here, their breakage propensity can be described as a function of their mechanical and physical properties and incident impact energy [2]. Several experimental approaches have been developed to assess particle breakability using various equipment, such as the single particle impact tester. The earliest one being the single particle impact tester developed by Yüregir et al. [3], where the size analysis is done gravimetrically by the sieving method. However, the device is not available commercially and therefore generally not accessible. Bonakdar et al. $[4,5]$ proposed a new approach, where the extent of particle breakage can be determined experimentally in a commerciallyavailable particle size analyser.

During pin milling, particles undergo a series of impacts at varying degree of impact velocities before they exit the milling chamber. The complex interaction between the particles and the pins is an important factor that decides the particle size distribution of the milled product. In this work, the particle dynamics and breakage behaviour of paracetamol crystals is analysed in a commercially available pin mill, PicoPlex using the Discrete Element Method (DEM). The particles are modelled as polyhedra. Particle energy spectra method in Rocky DEM software (see below) and the impact breakage model of Ghadiri and Zhang [2] are adopted to infer the breakage behaviour of particles in a pin mill. The effect of the process conditions such as the feed rate and rotational velocity of the pins on the breakage behaviour is investigated using such approach.

\section{Methodology}

The PicoPlex pin mill (50 mm diameter) of Hosokawa Micron (Fig. 1 (a)) is simulated using Rocky DEM software package (ESSS Co., Florianópolis, Brazil). For simulation, the material properties are based on paracetamol crystals. 327,388 and $461 \mu \mathrm{m}$ mono-sized

\footnotetext{
* Corresponding author: M.Ghadiri@ leeds.ac.uk

A video is available at https://doi.org/10.48448/0e7m-pk53
} 
particles corresponding to the geometric mean of sieve cuts of $300-355,355-425$ and $425-500 \mu \mathrm{m}$ (BS410 standard) are used. The shape of the particle is assumed as polyhedron of 26 faces that approximate the shape of a paracetamol crystal as shown in Fig. 1 (b). The geometry of the pin mill is simplified to remove features that would otherwise slow down the simulation (Fig. 1 (c)). The material properties and interactions used in the simulation are shown in Table 1. Feed rates of 0.2 and $0.4 \mathrm{~g} / \mathrm{s}$ are investigated as well as the rotational velocities (3000, 6000, 10000 and $30000 \mathrm{RPM}$ ) of the mill. $2 \mathrm{~s}$ of the process is simulated.
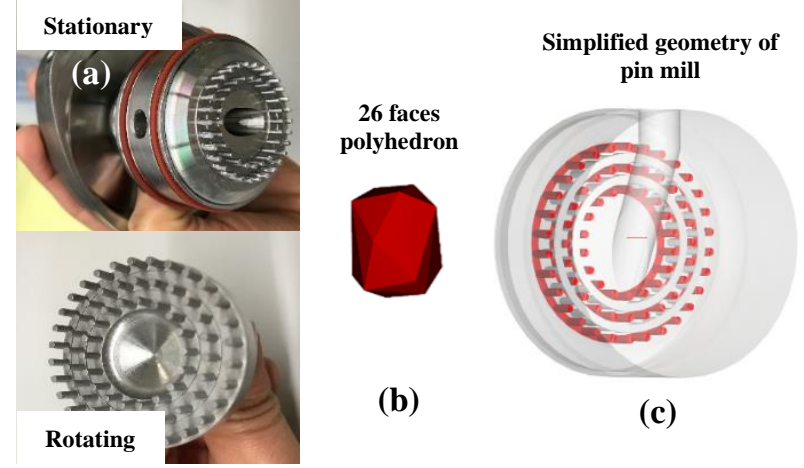

(c)

Fig. 1. PicoPlex pin mill of Hosokawa Micron comprising a stationary and a rotating part (a); particle modelled as 26 faces polyhedron (b) and simplified geometry of pin mill (c).

Table 1. Material properties and interactions.

\begin{tabular}{|c|c|c|}
\hline \multicolumn{3}{|c|}{ Material Properties } \\
\hline & Geometry & Particle \\
\hline Density, $\rho\left(\mathrm{kg} / \mathrm{m}^{3}\right)$ & 7800 & 1290 \\
\hline Young's Modulus, $E\left(\mathrm{~N} / \mathrm{m}^{2}\right)$ & $2 \times 10^{11}$ & $5.7 \times 10^{7}$ \\
\hline \multicolumn{3}{|c|}{ Material Interactions } \\
\hline & $\begin{array}{l}\text { Geometry- } \\
\text { particle }\end{array}$ & $\begin{array}{l}\text { particle- } \\
\text { particle }\end{array}$ \\
\hline static friction $(-)$ & 0.3 & 0.25 \\
\hline dynamic friction $(-)$ & 0.3 & 0.25 \\
\hline coefficient of restitution (-) & 0.5 & 0.25 \\
\hline
\end{tabular}

\section{Results and discussions}

\subsection{Average number of particle collisions}

The rotating disc of PicoPlex pin mill has three sets of rings radially placed so that for a set rotation speed they have three different linear velocities during operation (Table 2). As particles enter the milling chamber in the central region, they experience a series of impacts against the pins on both the stationary and rotating rings. The collisions of each individual particle in the simulation are tracked and recorded. The effect of rotational speed on the average number of particle collisions before they leave the mill is shown in Fig. 2 (327 $\mu \mathrm{m}$ particles fed at $0.2 \mathrm{~g} / \mathrm{s})$. It can be seen that the average number of particle collisions increases with the rotational velocity, but only against the middle and outer rings. At the inner ring, particle collision is the highest at 3000 RPM. This phenomenon can be attributed to the fact that the material feed rate used $(0.2$ $\mathrm{g} / \mathrm{s}$ ) is higher than the amount of particles that the inner ring can disperse properly at 3000 RPM, causing the particles to pile up at the inner ring and increases the collision detected. Similar behaviour is seen for the cases with bigger particle sizes (388 and $461 \mu \mathrm{m}$ ) and higher material feed rate $(0.4 \mathrm{~g} / \mathrm{s})$ but is not shown here for brevity.

Table 2. Linear velocity of the three rotating ring at different RPMs.

\begin{tabular}{|c|c|c|c|}
\hline \multicolumn{4}{|c|}{ Velocity } \\
\hline $\begin{array}{c}\text { Rotational } \\
(\mathbf{R P M})\end{array}$ & $\begin{array}{c}\text { Inner Ring, } \boldsymbol{V}_{\boldsymbol{i}} \\
(\mathbf{m} / \mathbf{s})\end{array}$ & $\begin{array}{c}\text { Middle Ring, } \boldsymbol{V}_{\boldsymbol{m}} \\
(\mathbf{m} / \mathbf{s})\end{array}$ & $\begin{array}{c}\text { Outer Ring, } \boldsymbol{V}_{\boldsymbol{o}} \\
(\mathbf{m} / \mathbf{s})\end{array}$ \\
\hline 3000 & 3.1 & 4.5 & 5.8 \\
\hline 6000 & 6.3 & 9.1 & 11.6 \\
\hline 10000 & 10.5 & 15.1 & 19.3 \\
\hline 30000 & 31 & 45 & 58 \\
\hline
\end{tabular}

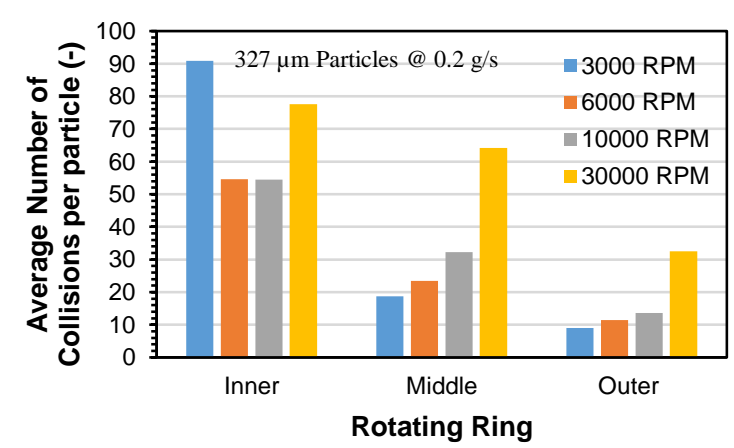

Fig. 2. Average number of collisions per particle at different rotating rings before they leave the mill.

\subsection{Collision energy spent on each rotating ring}

The cumulative dissipated power is calculated by summing up the dissipated energy for every collision (the difference between incident and rebound kinetic energy) for all the particles divided by the simulation time. This is shown in Fig. 3 as a function of the collision energy of a single $327 \mu \mathrm{m}$ particle for the three rings at $3000 \mathrm{RPM}$. Interestingly, despite having the largest number of collisions per particle, the inner ring does not have the highest expended energy; neither is the case for the outer ring, due to low collision frequency, albeit with the largest collision speed.

\subsection{Minimum energy for particle breakage}

Particle breakage is not simulated in this work, but the condition for breakage is analysed using a feature called "Energy Spectra" in the Rocky software. The incident kinetic energy associated with all collisions experienced by every individual particle in the system is recorded, thus producing a distribution for all the particle in the system. The values are expressed per unit mass (c.f. specific) and presented in cumulative form. If the minimum incident specific energy for a particle to break is known, the specific power for breakage can then be estimated and used to infer the breakage rate. In this work, the impact breakage model of Ghadiri and Zhang [2] is used to identify the minimum specific energy required to initiate particle breakage: $R^{*}=\alpha \frac{H}{K_{c}^{2}} \rho D v^{2}$, where $R^{*}$ is the fractional mass loss of particles due to impact, $\rho$ and $D$ are the particle density and size, and $v$ is impact velocity. The lumped parameter $\alpha H / K_{c}^{2}$ is known as the breakability 
index, in which $H$ and $K_{c}$ are hardness and toughness and $\alpha$ is the proportionality factor.

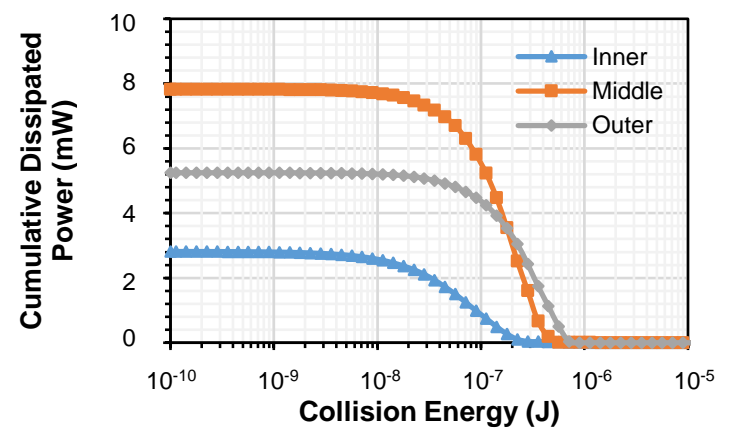

Fig. 3. Cumulative dissipated power of $0.4 \mathrm{~g}$ ( 34187 particles) of $327 \mu \mathrm{m}$ particles as a function of dissipated energy of single particles colliding with rotating rings at 3000 RPM.

The breakage of paracetamol obtained experimentally through single particle impact testing by Dogbe [6] is shown in Fig. 4, expressed as function of $\rho D v^{2}$. The intercept of the line with the abscissa represents the point where paracetamol particles start to break. Having this information, the transition velocity from no breakage to chipping of a given particle size can be determined.

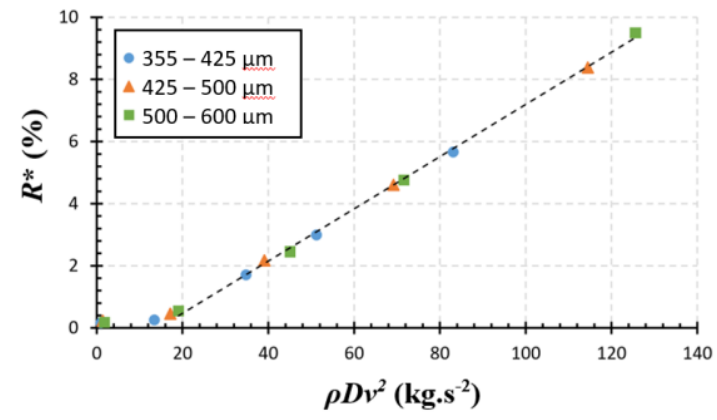

Fig. 4. Breakage of paracetamol crystals as a function of size and impact velocity, extracted from the work of Dogbe [4].

The minimum specific energy required for breakage, $E_{\min }$ is then obtained by simply converting the transition velocity, $V_{t}$ to specific kinetic energy using the equation: $E_{\min }=0.5 V_{t}^{2}$. This is shown in Figs 5 (a) and (b) as a solid vertical line, together with the particle energy spectra related to normal impact and shear of $327 \mu \mathrm{m}$ particles in a pin mill. The dashed vertical lines show the particle collision specific energy associated with the largest pin speed. The specific collision power is associated mainly with the normal component of impact velocity. The tangential velocity component, responsible for shearing is a much smaller component. In the case where the mill is operating at 6000 RPM (Fig. 5 (a)), the fraction of the particles exceeding minimum specific energy for breakage is expected to break. Further increasing the RPM increases the tendency of particles to break (Fig. 5 (b)). The power for particle breakage is found to increase with particle size (not shown for brevity), indicating that bigger particles break to a larger extent. Most of the collisions result in specific energy that is below the maximum specific energy, but there exists a fraction of collisions exceeding this limit. A plot of the distribution of particle impact velocities (against the inner ring) is shown in Fig. 6. This provides a clear indication that pin speed is not representative of the collision speed of all the particles.
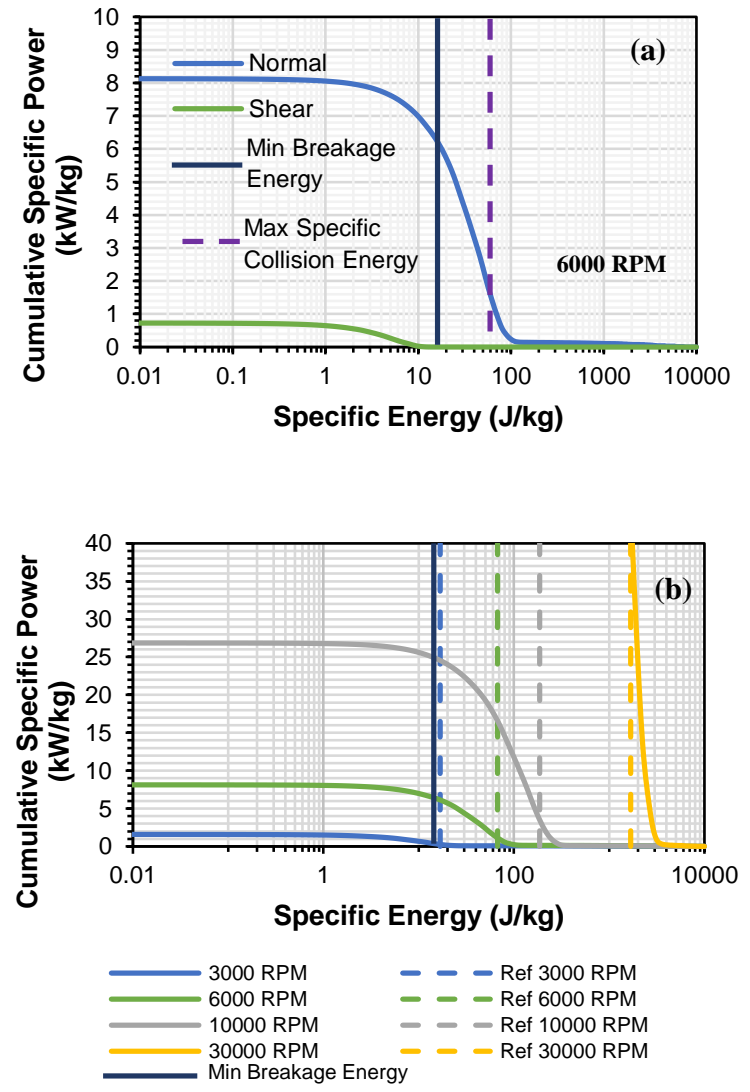

Fig. 5. Particle energy spectra of $327 \mu \mathrm{m}$ particles; (a): normal impact and shear in a pin mill rotating at 6000 RPM; (b): normal impact only at different RPMs. The solid and dashed vertical lines represent the minimum breakage energy of paracetamol particles and the maximum incident kinetic energy for $327 \mu \mathrm{m}$ particle size and the given RPM. $N B$ : the energy spectra curve for 30000 RPM is trimmed off from the top to better show the difference amongst different RPM.

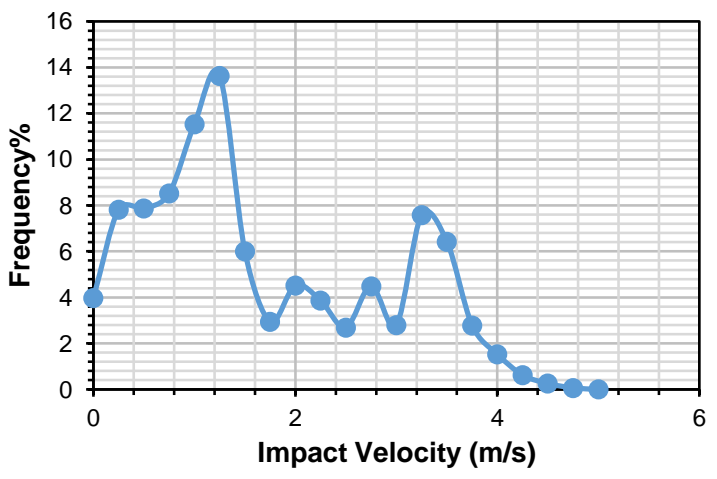

Fig. 6. Distribution of impact velocities for $425-500 \mu \mathrm{m}$ particle against inner ring at 3000 RPM.

\subsection{Particle breakage}

Impact is known to be the dominant breakage mechanism in a pin mill and this is in agreement with the results 
shown previously (Fig. 5(a)). A very interesting phenomenon is observed in the simulation cases of 461 $\mu \mathrm{m}$ particles. Operating at $3000 \mathrm{RPM}$, the energy spectrum of the particles shows unexpectedly high collision energy as shown in Fig. 7 (a). The light dashed line represents the specific energy associated with particle collision with the outer ring (i.e. largest speed) at a given RPM. It gives an indicative value for the energy level expected of the particles when the mill is operating at 3000 RPM. In the cases where particles are smaller in size (327 and $388 \mu \mathrm{m}$ ), most of the specific energies recorded are always below the specific reference given (not shown for brevity). However, as shown in Fig. 7 (a), the highest energy recorded is well above the maximum specific energy for 3000 RPM, clearly implying again that the pin speed is not a good indicator of representative collision velocity.

A careful investigation also reveals that particles of $461 \mu \mathrm{m}$ in size have a tendency to get nipped between the rotating rings and stationary rings. Consequently, the particles experience very high stresses and therefore this causes an increase in the specific energy recorded. This signifies the importance of the particle size and gap size when designing a milling operation and will affect the prediction of particle breakage due to a change in the stressing mode. Nipping in small clearances in mixing by impellers has been reported in literature [7] and here we show for the first time it also prevails in pin milling. Such behaviour is very common in impeller-agitated powder beds, where big particles with diameters approaching the size of the gap between the impeller and vessel are used. Substantial shear stressing due to nipping could occur when the clearance size to particle diameter ratio is less than 2.5. Interestingly, such phenomenon is not seen when the mill is operating at higher rotational speeds. The particle energy spectrum for the mill operating at 10000 RPM is shown in Fig. 7 (b). The distribution is well below the maximum specific energy corresponding to the outer ring speed of the pin mill operating at $10000 \mathrm{RPM}$, but its high tail end exceeds this value. The fact that at high rotational speeds such behaviour is not seen could be attributed to particles getting quickly dispersed by the rotating rings. At low RPM, particles tend to accumulate between the inlet and the inner ring, causing them to pile up. That in turn increases the chance of particles getting nipped by the rings, giving rise to unexpectedly high energy levels shown in Fig. 7(a).

\section{Conclusions}

The particle dynamics in a rotating pin mill was analysed. It was found that most particle collisions are against the inner rotating ring, which is running at the lowest speed. Increasing the rotational speed increases the average number of particle impacts. While having the largest number of collision per particle, the energy expended at the inner rotating ring is the lowest. The highest impact energy is recorded at the outer ring, but most of the energy is actually dissipated at the middle rotating ring. Therefore, the pin speed does not correlate with particle impact velocity. This is important as the analysis of the operation of pin mills and scaling up are commonly based on the pin speed. This could lead to underestimation of particle breakage extent. Particle Energy Spectra method was used in conjunction with mechanistic impact breakage model of Ghadiri and Zhang [2] to infer the breakage behaviour in a pin mill. Particle nipping was found for the largest particle size, i.e. for $461 \mu \mathrm{m}$ particles as this is approaching the gap size between the rings $(\sim 500 \mu \mathrm{m})$. This behaviour was not seen when the rotational speed was increased to 10000 RPM or higher.
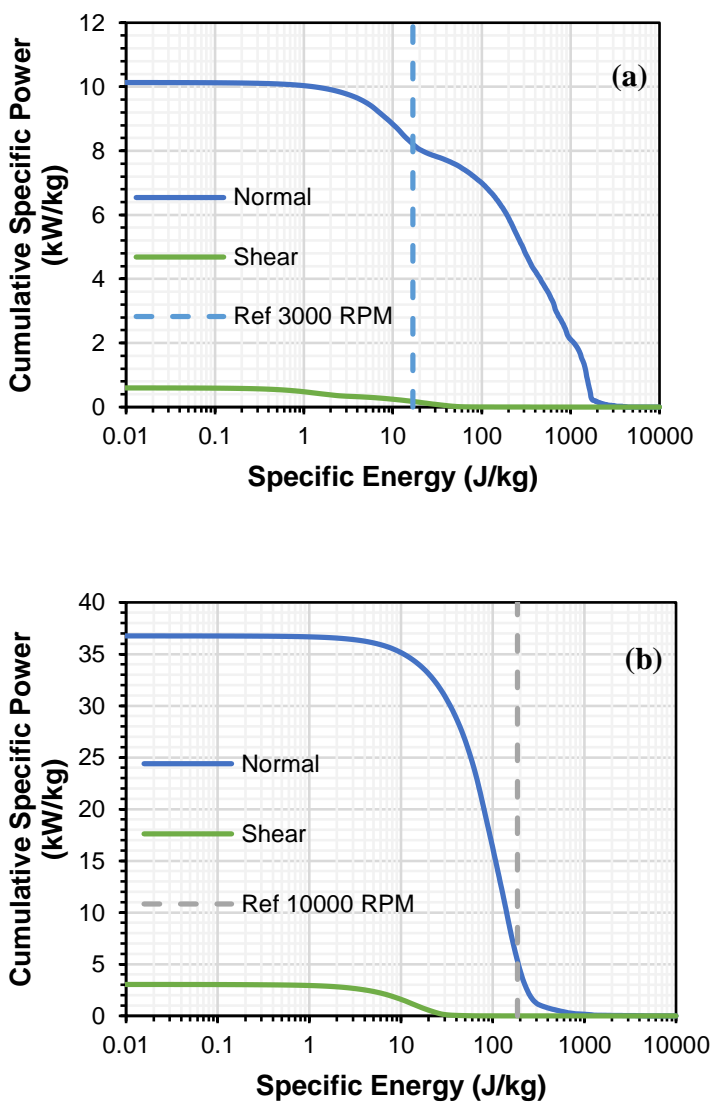

Fig. 7. Particle energy spectra curves of $461 \mu \mathrm{m}$ particles in a pin mill rotating at (a) 3000 RPM and (b) 10000 RPM

The authors gratefully acknowledge the support of the Advanced Manufacturing Supply Chain Initiative through the funding of the 'Advanced Digital Design of Pharmaceutical Therapeutics' (Grant No. 14060) project within which the work reported was conducted.

\section{References}

[1] R. Rowe. Handbook of Pharmaceutical Excipient (2006)

[2] M. Ghadiri, Z. Zhang. Chem. Eng. Sci. 57, 36593669 (2002)

[3] K.R. Yüregir, M. Ghadiri, R. Clift. Powder Technol. 42, 843-853 (1987)

[4] T. Bonakdar, M. Ali, S. Dogbe, M. Ghadiri, A. Tinke. Int J Pharm. 501, 65-74 (2016)

[5] T. Bonakdar, M. Ali, M. Ghadiri. Int J Pharm. In press (2021)

[6] S. Dogbe. Predictive Milling of Active Pharmaceutical Ingredients and Excipient. (PhD Thesis - 2016)

[7] C. Crutchley, J. Bridgwater. KONA Powder and Particle Journal. 15, 21-31 (1997) 